\title{
Nanoscale
}

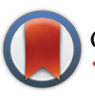

CrossMark \& click for updates

Cite this: Nanoscale, 2016, 8, 20037

\section{Clustered localization of EGFRvIII in glioblastoma cells as detected by high precision localization microscopy}

\author{
Philip S. Boyd,$\uparrow^{\mathrm{a}}$ Nina Struve, ${ }^{*} \dagger^{\mathrm{b}}$ Margund Bach, ${ }^{\mathrm{a}}$ Jan Philipp Eberle, ${ }^{a}$ Martin Gote, ${ }^{a}$ \\ Florian Schock, ${ }^{\mathrm{c}}$ Christoph Cremer, ${ }^{\mathrm{a}, \mathrm{c}}$ Malte Kriegs $\dagger^{\mathrm{b}}$ and Michael Hausmann* ${ }^{a}$
}

For receptor tyrosine kinases supramolecular organization on the cell membrane is critical for their function. Super-resolution fluorescence microscopy techniques have offered new opportunities for the analysis of single receptor localization. Here, we analysed the cluster formation of the epidermal growth factor receptor variant III (EGFRvIII), a deletion variant which is expressed in glioblastoma. The constitutively activated variant EGFRvIII is expressed in cells with an egfr gene amplification and is thought to enhance the tumorigenic potential especially of glioblastoma cells. Due to the lack of an adequate model system, it is still unclear how endogenous EGFRvIII expression alters cellular signalling and if it is organized in clusters like the wild type receptor. We have recently described the establishment of two pairs of isogenetic cell lines (BS153 and DKMG), displaying endogenous EGFRvIll expression or not. Using these cell lines we investigated single receptor localization of EGFRvIII by high precision localization microscopy. Cluster analysis revealed that EGFRvIII is present in clusters on the surface of the cells, with about $60 \%$ or even more receptor molecules being assembled in clusters of approximately $100 \mathrm{~nm}$ in diameter whereby the cluster definition was iteratively determined. The signal to signal distance may indicate dimer formation while signal quantification indicates $1 \times 10^{6}-5 \times 10^{6}$ EGFRvIII molecules per cell. Altogether, these data give unique insights into the membrane surface localization of EGFRvIll in glioblastoma cells. These insights will help to unveil the function of this tumour associated receptor variant which might lead to a better understanding of glioblastoma and therefore could lead to improved therapy approaches.

Received 25th July 2016 , Accepted 8th November 2016

DOI: $10.1039 / c 6 n r 05880 a$

www.rsc.org/nanoscale often associated with poor prognosis, ${ }^{2}$ understanding of mechanisms behind these survival pathways would help to improve anti-EGFR therapies. ${ }^{3}$

Physical simulation models ${ }^{4,5}$ of receptor proteins embedded in lipid bi-layers have predicted receptor cluster formation due to liquid forces and hydrophobic mismatch. ${ }^{6-8}$ With the development of super-resolution light microscopy and near-field optical microscopy it has become feasible to detect such cluster formations for several receptors and to measure their size and molecular compartmentalization in the sub-diffraction range, i.e. in the resolution range of single molecules, a range of a few ten nanometres. ${ }^{9-12}$

These nano-structured cluster units lead to functional consequences for inter-receptor communication and signalling pathways. ${ }^{13}$ In addition many of these receptor units are often associated with membranous micro-domains such as lipid rafts, caveolae and coated pits and thus provide spatial and temporal control of signalling mechanisms. Therefore mechanistic understanding of EGFR over-activation requires not only the knowledge of presumptive signalling pathways but especially the knowledge of spatial organization of the cell surface.

\footnotetext{
${ }^{a}$ Kirchhoff-Institute for Physics, University of Heidelberg, Im Neuenheimer Feld 227, 69120 Heidelberg; Germany. E-mail: hausmann@kip.uni-heidelberg.de; Fax: [+49] 622154 9112; Tel: [+49] 6221549824

${ }^{b}$ Laboratory of Radiobiology \& Experimental Radiooncology, University Medical Center Hamburg - Eppendorf, Martinistr. 52, 20246 Hamburg, Germany. E-mail: ni.struve@uke.de; Fax: [+49] 407410 55139; Tel: [+49] 40741053597 ${ }^{c}$ Institute for Molecular Biology, Ackermannweg 4, 55128 Mainz, Germany $\dagger$ Equal contribution.

$\$$ Equal contribution.
} 
The epidermal growth factor receptor variant III (EGFRvIII) $^{14}$ is a deletion variant of the RTK EGFR, which lacks the exons 2-7. The EGFRvIII is frequently expressed in glioblastoma multiforme (GBM), which is the most common malignant brain tumour in adult patients, with an estimated 5 -year survival rate of less than $10 \% .{ }^{15,16}$ The expression of EGFRvIII is associated with the amplification of the egfr gene and increased wild type (WT) EGFR expression. ${ }^{17}$ EGFRvIII has been shown to be an important factor in driving tumour progression and is correlating with poor prognosis (for review see ref. 14).

In contrast to the wt EGFR, which is activated by ligand binding and subsequent dimerization, EGFRvIII is thought to be constitutively active. ${ }^{18}$ Due to the lack of an adequate model system, the precise mechanisms of this activation and the regulated signalling pathways are not completely understood; however, even dimerization independent activity has been described. ${ }^{19}$ The wt EGFR is known to be localized in membranous structures such as coated pits ${ }^{9}$ and super-resolution imaging has revealed clustering of EGFR in a lipid raft dependent manner. ${ }^{12}$ However, less is known about the precise localization of EGFRvIII and due to the lack of an appropriate model system, it is still an open question, whether it also forms clusters or not. Whereas wt EGFR is known to undergo homo- and hetero-dimerization, resulting into an enhanced kinase activity, the formation of homo- and /or hetero-dimers of EGFRvIII is still a subject of investigation. ${ }^{20}$ Although the precise functions and consequences of EGFRvIII (over) expression and the spatial arrangements and dimerization potential are not fully understood, this RTK is a promising target in GBM treatment. ${ }^{3}$ This motivates detailed studies of functional mechanisms.

We have recently reported the establishment of an EGFRvIII+/- model system consisting of two iso-genetic cell pairs based on the endogenously EGFRvIII expressing BS153 and DKMG glioblastoma cell lines. For both cell lines we generated iso-genetic sublines with either a very high amount of EGFRvIII expressing cells (BS153vIII+ and DKMGvIII+) or a very low amount of EGFRvIII expressing cells (BS153vIII- and DKMGvIII-). ${ }^{21}$ To gain more information about the spatial organization with respect to regulation of the EGFRvIII, we analysed these sublines by high precision localization microscopy ${ }^{22}$ in order to further quantify presumptive cluster formation.

To analyse receptor complexes by fluorescence light microscopy, the proteins have to be specifically labelled with fluorophore tagged antibodies, which can be imaged using multi-colour fluorescence microscopy setups. However, a serious impediment to exploit the full potential of light microscopy has been the diffraction limited optical resolution described by the Abbe-Rayleigh limit. ${ }^{23,24}$ With high numerical aperture objective lenses this resolution limit approaches about $200 \mathrm{~nm}$ laterally and about $600 \mathrm{~nm}$ axially in biological specimens. Thus, an ensemble of fluorescently labelled molecules like proteins would lead, due to point spreading, to more or less homogeneously overall stained images of cells.
Novel approaches in light microscopy circumventing the Abbe-Rayleigh boundary conditions, however, enable effective optical resolutions down to the order of $10 \mathrm{~nm}$ or even better. ${ }^{25}$ One of these methods using standard objective lenses is localization microscopy ${ }^{22}$ based on the fundamental concept of optical isolation by different spectral signatures, e.g. by using constant differences in the absorption/emission spectrum $^{26}$ or fluorophores that can be switched between two different spectral states [see for instance PALM ${ }^{27}$ (F)PALM, ${ }^{28}$ STORM,${ }^{29}$ dSTORM, ${ }^{30}$ SPDM $_{\text {phymod }}{ }^{31}$ GSDIM $^{32}$ etc.] to achieve a temporal isolation and thus a spatial separation of single signals. This allows the determination of signal positions (spatial coordinates) and their spatial distances even if they are adjacent below the Abbe-Rayleigh resolution limit. All acquired positions of individual fluorescent molecules can then be merged into one artificial, pointillist, super-resolution image, in which the effective resolution is depending on the lateral and axial localization precision. ${ }^{33,34}$

A certain embodiment of localization microscopy used in the experiments described here, is SPDM (Spectral Position Determination Microscopy ${ }^{31,35}$ ), having the advantage of using conventional fluorophores which are switched to a "dark" state by laser light-induced reversible photo bleaching. ${ }^{36,37}$ From this dark state the fluorescent molecules statistically fall back into the emission state. Each of the emitting fluorophores is represented by an Airy disc in the microscopy image. The centre-of-mass (barycentre) of such a disc approximates to a high accuracy the location of the emitting molecule. With a typical localization precision and the point-to-point distance resolution of SPDM images in the order of 10-20 nm, pointillist images locally differing in point densities provide structural resolution based on the detected molecule positions. Thus, receptor cluster formation, ${ }^{10}$ chromatin re-arrangements, ${ }^{38}$ protein trafficking ${ }^{39}$ and chromatin loop formation ${ }^{40}$ have for instance been studied by SPDM.

\section{Results and discussion}

\section{EGFRvIII expression in vIII+ and vIII- sublines}

The goal of this study was to analyse the supramolecular organization of EGFRvIII. To this end we used two iso-genetic pairs of GBM sublines, the BS153vIII- and BS153vIII+ and the DKMGvIII- and DKMGvIII+ sublines. ${ }^{21}$ Both EGFRvIII+ sublines displayed more than $80 \%$ of EGFRvIII expressing cells while the EGFRvIII- sublines showed less than 1\% EGFRvIIII expressing cells as measured by flow cytometry using the EGFRvIII specific antibody L8A4 (Fig. 1A). Western blot analysis of these cells did also not reveal EGFRvIII expression in EGFRvIII- sublines while strong expression in the EGFRvIII+ sublines was observed. EGFRvIII expression was associated with a stronger expression of wt EGFR, although this in general was more pronounced for the BS153 cells (Fig. 1B). $3 \mathrm{D}$ immunofluorescence imaging revealed membranous EGFRvIII, with a higher intensity for the BS153vIII+ compared to the DKMGvIII+ cells, indicating a higher amount of fluores- 
A

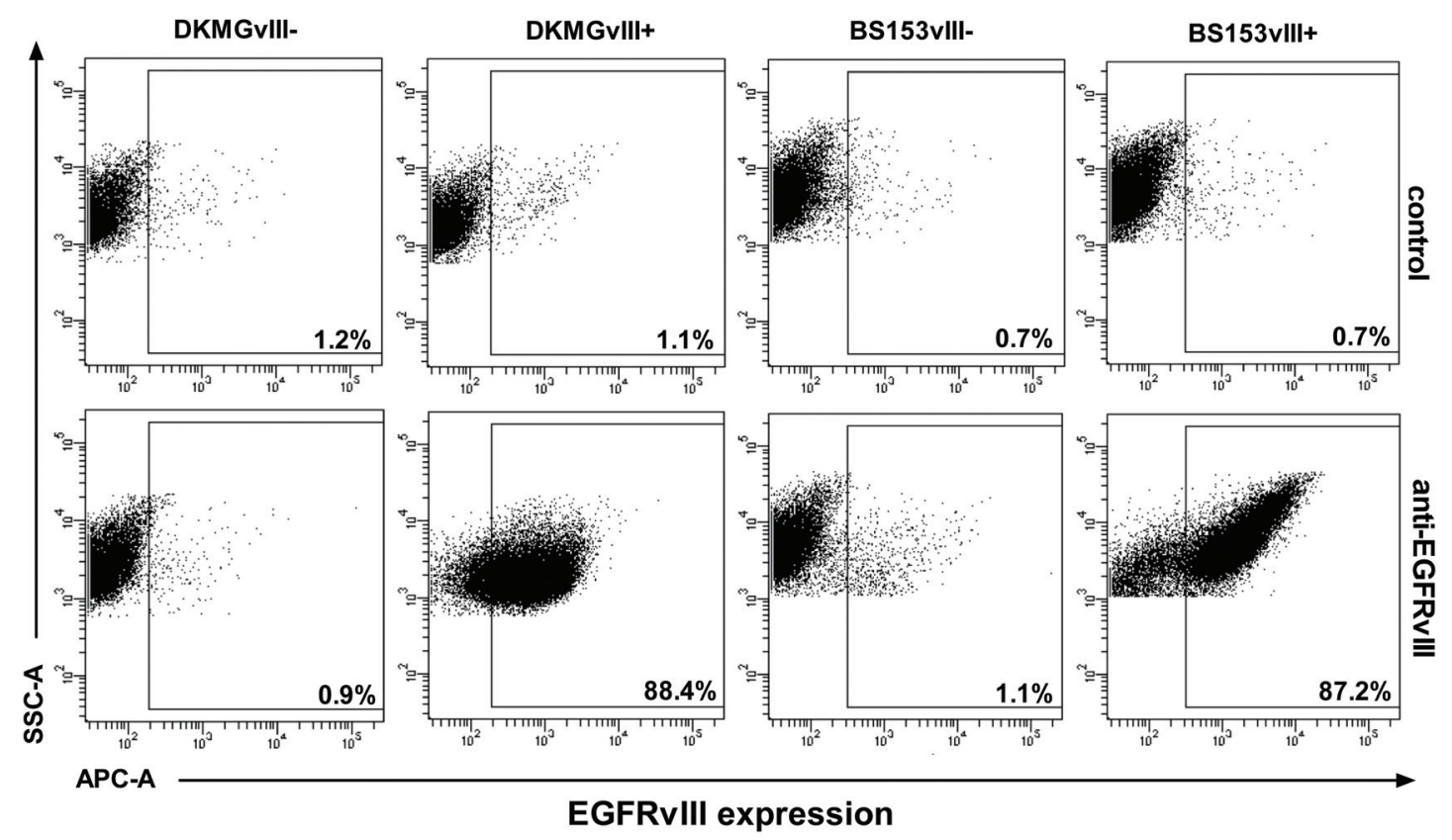

B

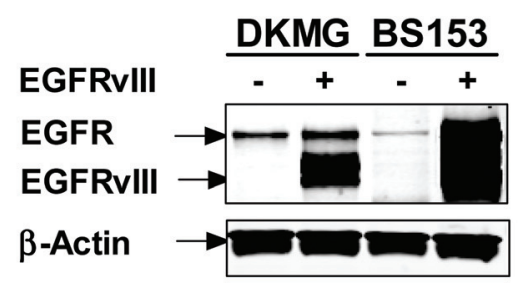

C
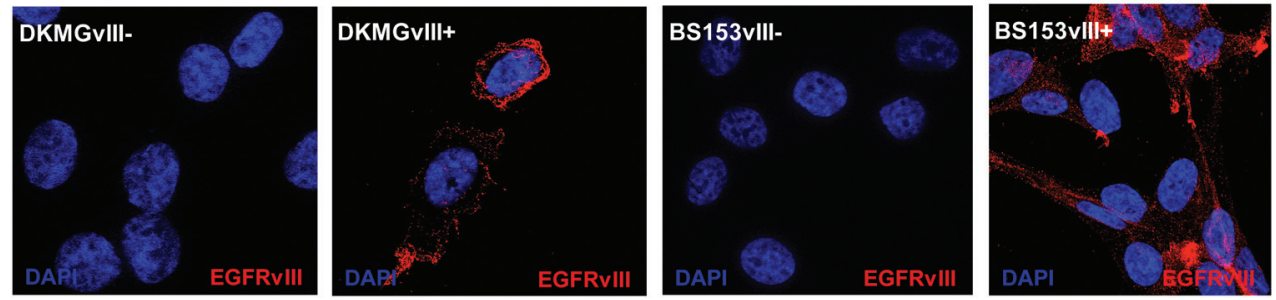

Fig. 1 EGFRvIll expression in EGFRvIII+/- GBM sublines. (A) Flow cytometry measurements: side scatter signal (SSC-A) vs. fluorescence intensity (APC-A) of the ALEXA stained secondary antibody against the primary antibody L8A4. Lower panel of dot-plots: the BS153vIII+ and DKMGvllI+ sublines displayed more than $80 \%$ of EGFRvlll expressing cells, vlll- sublines less than $1 \%$. Upper panel of dot-plots: control cells were stained without the primary antibody. They only show a scatter signal with negligible fluorescence. (B) EGFR and EGFRvIll expression detected by western blotting in EGFRvIII+ and EGFRvIII- sublines using a pan-EGFR antibody (C) EGFR and EGFRvIll expression detected by immunofluorescence in EGFRvIII+ and EGFRvIII- sublines using the EGFRvIll specific L8A4 antibody. The strong red signal on the cytoplasm membrane indicates the degree of expression: very strong BS153vIII+, strong DKMGvIII+, no BS153vIll-, and no DKMGvIll-. The cell nuclei were counterstained with DAPI.

cently labelled EGFRvIII molecules in the cellular membrane per cell (Fig. 1C). This higher EGFRvIII expression per cell is supported by the western blot and also by the flow cytometry data (Fig. 1A and B).

These data indicate that the two EGFRvIII overexpressing cell lines differ in their degree of overexpression. However, western blotting as well as flow cytometry, although analysing single cells, display only average differences between the cell lines without considering intracellular variations. This can partially be overcome by light microscopy on the micro-scale where staining intensity represents the relative expression level in distinct areas or compartments of the cell. Nevertheless, 
due to point spreading molecular arrangements of antibody labelled molecules cannot be elucidated. This, however, requires a more sophisticated approach as shown in the following chapters.

\section{EGFRvIII detection using high precision localization microscopy}

To study the supramolecular organization and spatial arrangements of EGFRvIII receptors in the membrane of GBM cells we used high precision localization microscopy (SPDM). ${ }^{31,35,41}$ EGFRvIII was stained in BS153vIII+/BS153vIII- and DKMGvIII+/DKMGvIII- cells respectively, using the specific L8A4 antibody and an ALEXAfluor® 594-labeled anti-mouse antibody for molecular tagging. For control experiments indicating fluorescence caused by non-specific antibody attachment, only the secondary antibody was added to specimens. The localization of antibodies labelling the EGFRvIII receptors was determined on the cell membrane in flat regions of the cell in order to avoid image plane shifts due to the cell nucleus.

In Fig. 2 an example of localization images is shown. At a first glimpse by visual inspection there seems to be a different point distribution between the specifically labelled specimens and the controls. Specific labelling revealed a non-homogeneous distribution of EGFRvIII on the cell membrane;
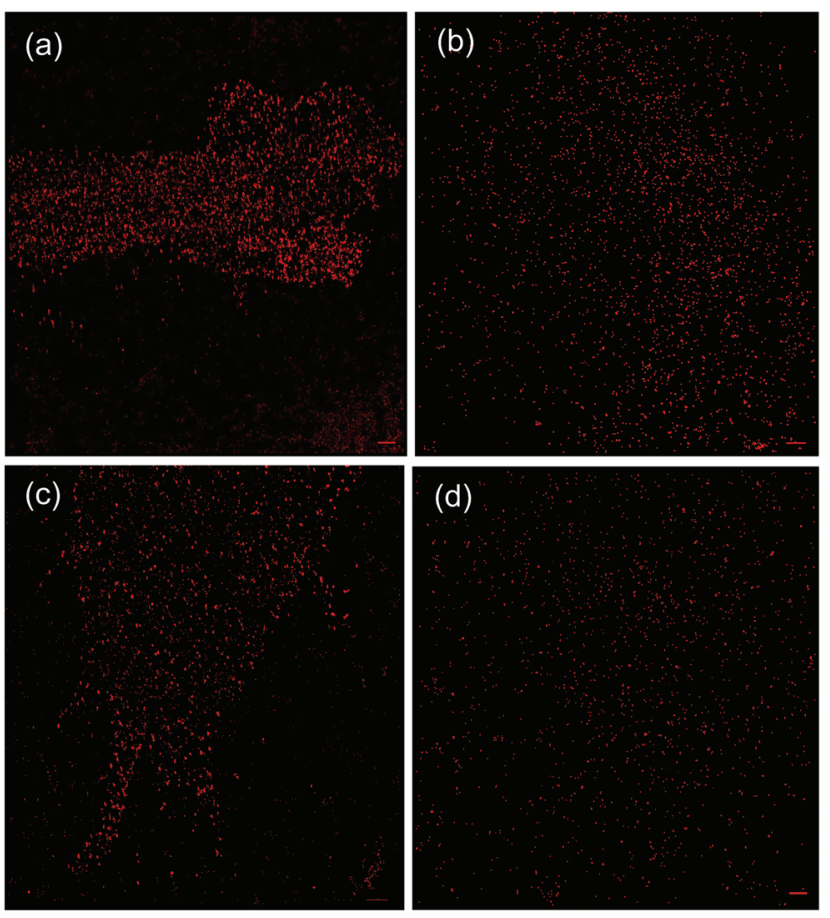

Fig. 2 Examples of typical localization images: cell membrane section of a BS153vllI+ cell (a) and a DKMGvllI+ cell (c) labelled with the specific EGFRvIII primary and the fluorochrome tagged secondary antibody; cell membrane section of a BS153vllI+ cell (b) and a DKMGvIII+ cell (d) of the control experiments where only the secondary antibody was added to the cell specimen. Scale bar $1 \mu \mathrm{m}$. regions of higher and lower densities were visible. In total the number of points seems to be increased.

For further quantification the orte-matrices were evaluated and different representations of the reconstructed images were possible as shown in Fig. 3. In the wide field image underlaying Fig. 3a and e, intensity fluctuations are due to the standing wave-field option of the instrument (see the Experimental section). These fluctuations do not occur in the pointillist localization image since the localization mode is taking a different optical beam path. The reconstructed localization image obtained from an image stack of about 1000 consecutively acquired images can be over-laid (Fig. 3c). In Fig. 3d a cluster image is shown (for details see next chapter) where each cluster is represented by another colour. In Fig. 3e an example for a nearest-neighbour image is shown. In this representation the brightness of an image point represents the relative number on next neighbours. In contrast to the cluster distribution which neglects those points that were not parts of a cluster, the nearest-neighbour image does still contain all image points.

These images demonstrate that we were able to detect EGFRvIII in the membrane of GBM cells using high precision localization microscopy and that data evaluation according to different classification parameters was possible. In the case of EGFRvIII overexpression about 34000 fluorescent signals were on average detected within a ROI of BS153vIII+ cells and about 21000 within a ROI of DKMGvIII+ cells. A rough estimate based on the assumption of a more or less homogeneous signal distribution indicated that about $2 \times 10^{6}-5 \times 10^{6}$ molecules could be found on the membrane of BS153vIII+ cells and about $2 / 3$ on the DKMGvIII+ cells, which was in good agreement with recently published estimates for other cell lines. ${ }^{42}$

\section{EGFRvIII is expressed in clusters on the cell surface of GBM cells}

In order to test whether EGFRvIII is organized in specific cluster formations, the receptor localizations were recorded by the position of the fluorochrome molecules of the antibody labelling system. These localization coordinates were obtained as the intensity bary-centres and represented by the ortematrix for further evaluation.

For this evaluation a cluster criterion was iteratively determined. Based on our own experience with ErbB2-receptor arrangements, ${ }^{10}$ a starting configuration of 5 neighbours within a radius of $60 \mathrm{~nm}$ for a minimal cluster was set. The cluster radius and the number of next neighbours were varied for a higher and a lower point density, i.e., for a higher point density a radius of $50 \mathrm{~nm}$ with 5 next neighbours and a radius of $60 \mathrm{~nm}$ with 6 next neighbours, and for a lower point density a radius of $60 \mathrm{~nm}$ with 4 next neighbours and a radius of $70 \mathrm{~nm}$ with 5 next neighbours.

In Fig. 4 the relative frequency of distances within (top row) and outside the clusters (bottom row) are shown for the different cluster parameters. These data sets were compared to a random distribution of the same number of points. The histograms show a significant increase of short distances 


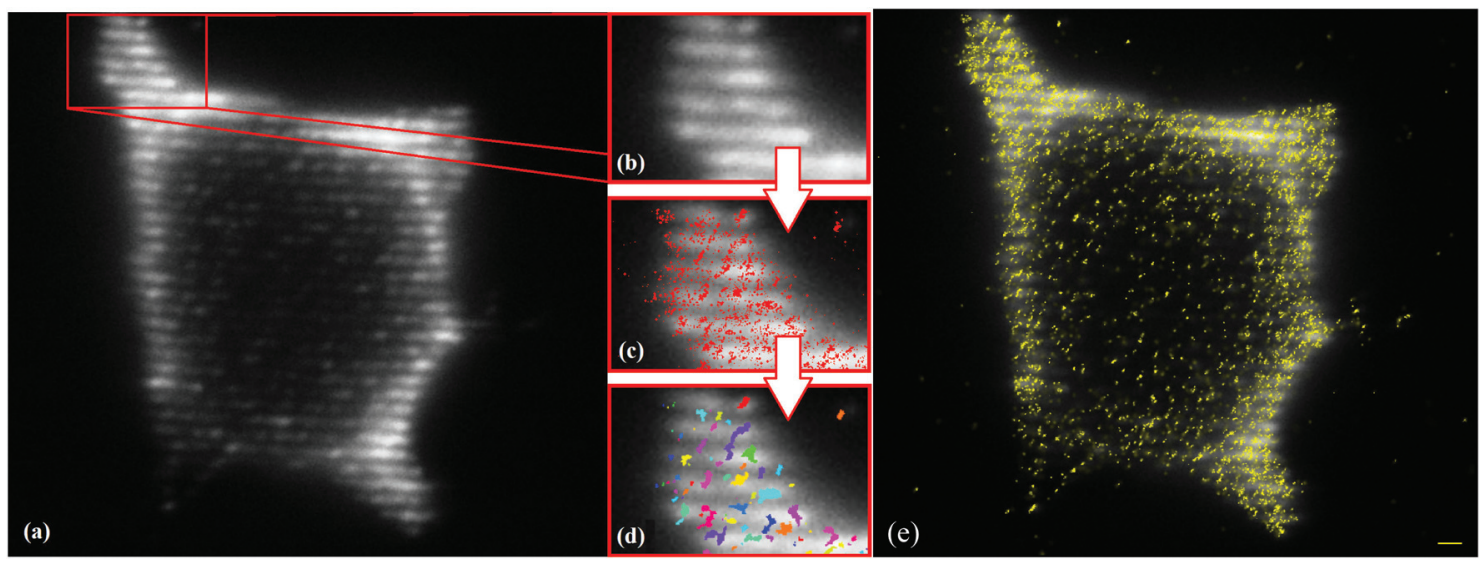

Fig. 3 Detection of EGFRvIll using high precision localization microscopy. (a) Wide-field image of the labelled receptors on the cell membrane; (b) image section of a typical ROI; (c) merged image of the wide-field image (grey) and the pointillist localization image (red); (d) cluster representation of the points involved in clusters; (e) merged image of the wide-field image (grey) and the nearest-neighbour image (yellow). Scale bar $1 \mu \mathrm{m}$.

within the cluster radius. For larger distances the localization data outside the clusters follow the random distribution. In the histograms of higher point densities (Fig. 4A), the distribution of shorter distances is more pronounced and smaller. However outside the clusters the deviation from the random distribution is larger. In contrast for lower point densities (Fig. 4B), these distributions follow the random histogram but the peak for shorter distances within the clusters is less pronounced and broadened. This means that many points are referred to be within a cluster although the point density is low. Consequently, too strict conditions would lead to only a few clusters and exclude obvious cluster formations, whereas less strict conditions include randomly distributed points into larger clusters. Thus the selection of minimum 5 points within a radius of $60 \mathrm{~nm}$ ((b) in Fig. $4 \mathrm{~A}$ and $\mathrm{B}$ ) appears to be the optimum compromise.

In Fig. 5 the results for cluster determination of an image section from the DKMGvIII+ cell line (Fig. 5a) are shown in comparison to the DKMGvIII- cell line (Fig. 5b). The chosen parameters fit the visual inspection very well: for DKMGvIII+ cells, the number of clusters and the number of points are significantly higher than those for DKMGvIII-. Although an increase of the cluster radius would increase the number of points within such a cluster, it would not describe an accumulation of points, i.e. a locally increased point density. For values chosen much higher than the initial cluster definition, it is obvious that such a parameter choice would not fit an intuitive cluster definition (Fig. 5c).

According to the selected cluster parameters the percentage of all signals found in clusters were determined for the cell types analysed. For the EGFRvIII+ cells about $60 \%$ or even more of the signals were allocated to clusters. In contrast the EGFRvIII- cells showed about $20 \%$ or even less points following the cluster criterion. For the BS153vIII- cells this was in the range of the control cells indicating that about $10 \%$ points in clusters is compatible with a random distribution of the points. These results clearly verify that EGFRvIII is forming clustered spatial arrangements in the case of being (over) expressed.

The clusters were statistically analysed for distances in clusters (in $\mathrm{nm}$ ), cluster size (= number of points detected in the individual clusters), cluster diameters (approximated by a circle in $\mathrm{nm}$ ), and point densities in the clusters (detected points per $\mu \mathrm{m}^{2}$ ). Fig. 6 shows some typical examples for frequency histograms.

In Table 1 the results of the cluster analysis are summarized. For the EGFRvIII- cell lines the mean number of signals in clusters is about the same and on the level of the control specimens. For BS153vIII+ the number of signals found in clusters is slightly increased as compared to DKMGvIII+. This might reflect the difference in EGFRvIII activation and expression between the two cell lines and is supported by the cluster size/diameter values. In addition, western blot analyses revealed a different EGFR and EGFRvIII phosphorylation in these EGFRvIII+ cell lines indicating different activities (data not shown).

There is also a slight increase of the mean percentage of signals found in clusters in DKMGvIII- cells as compared to the control or BS153vIII- cells. This might indicate that some cells of DKMGvIII- might still show some EGFRvIII expression.

Measuring the distances between the signals indicates the internal structure of the clusters. Comparing the histograms shows that the maximum is at about $25 \mathrm{~nm}$ with the exception of BS153vIII+ cells where it is at $45 \mathrm{~nm}$. This is compatible to the increase of the mean diameter of the BS153vIII+ clusters and the decrease of the molecular density which usually indicates an enhanced activity as known from other receptors of the EGF-family. ${ }^{43}$ This is in line with the enhanced activity of downstream signalling pathways observed for the BS153vIII+ cells compared to the DKMGvIII+ cells. ${ }^{21}$

Moreover, the increase of cluster size associated with a decrease of signal density may give a hint of homo- and/or heterodimerization of EGFRvIII since the so far applied labelling strategy of a specific EGFRvIII antibody and a fluorescent 

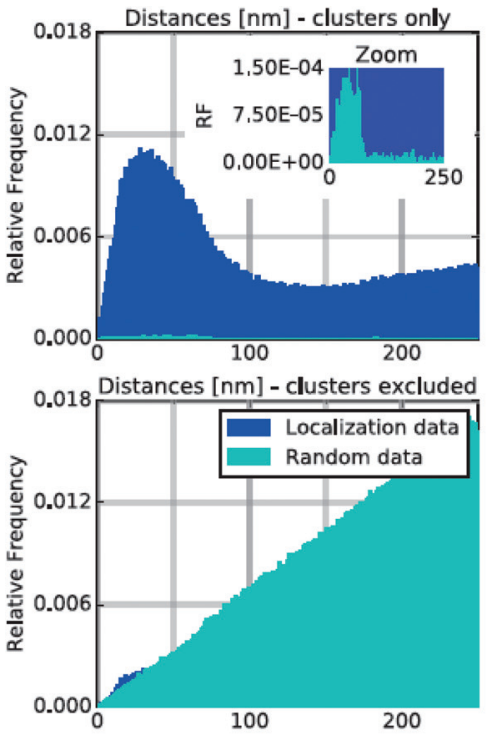

(A) $70 \mathrm{~nm}, 5 \mathrm{NN}$

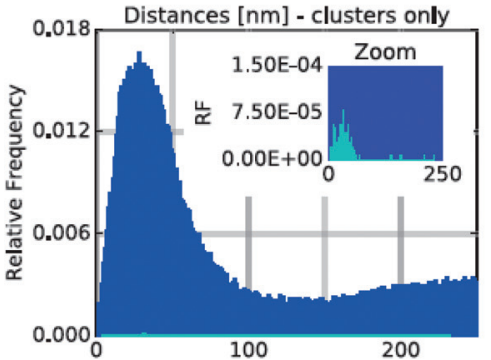

0.018 Distances [nm] - clusters excluded

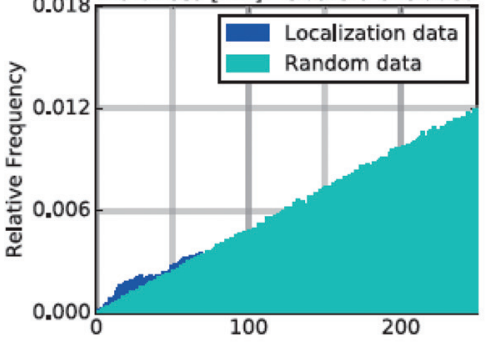

(D) $50 \mathrm{~nm}, 5 \mathrm{NN}$
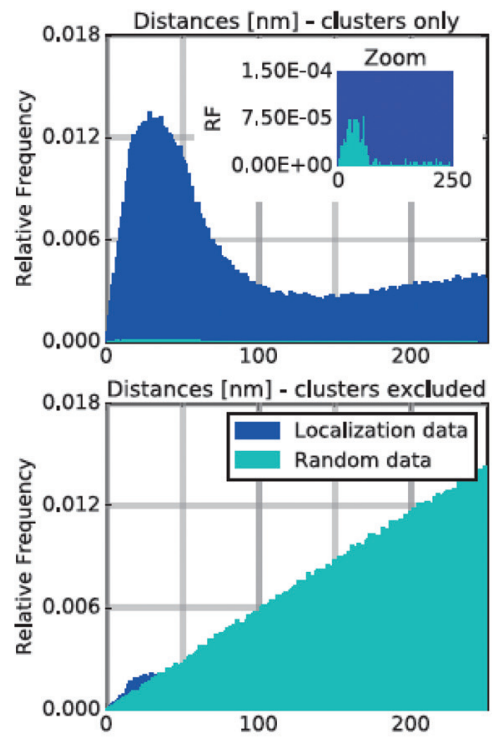

(B) $60 \mathrm{~nm}, 5 \mathrm{NN}$

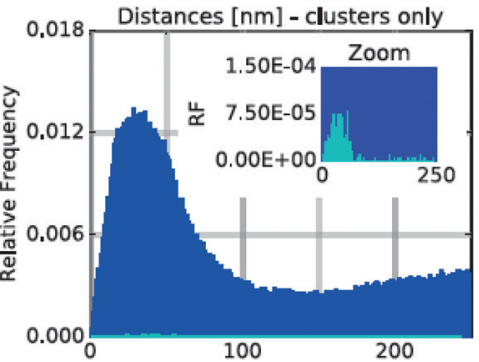

0.018 Distances [nm] - clusters excluded

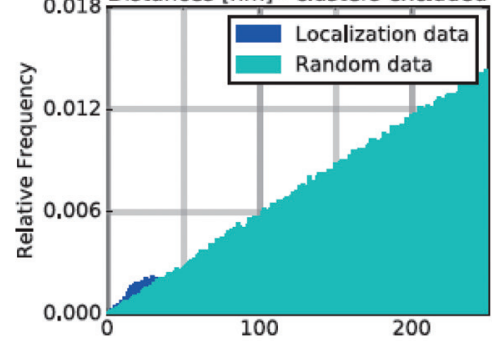

(E) $60 \mathrm{~nm}, 5 \mathrm{NN}$
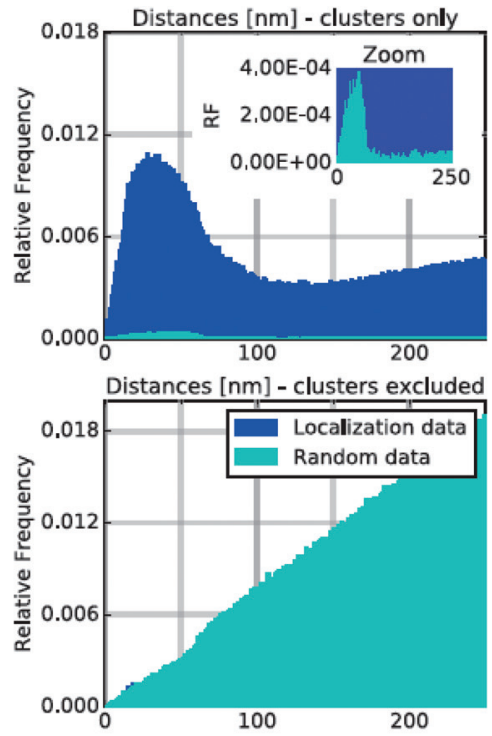

(C) $60 \mathrm{~nm}, 4 \mathrm{NN}$
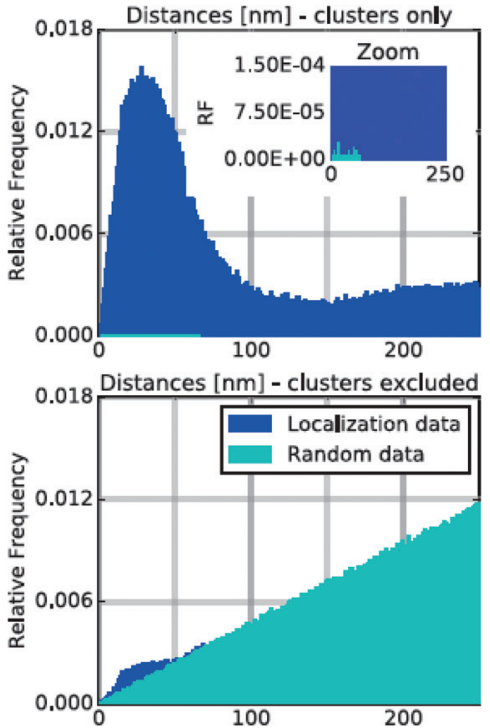

(F) $60 \mathrm{~nm}, 6 \mathrm{NN}$

Fig. 4 Iterative determination of the cluster parameters. Distance frequency histograms for points within and outside the defined clusters in comparison to random distributions with the same point numbers. $(A-C)$ Distributions with higher point densities, (D-F) distributions with lower point densities in comparison to 5 next neighbours within a radius of $60 \mathrm{~nm}$. For comparison with random data, a zoomed image is inserted.

second antibody would result in one signal point for dimers because heterodimers would further require another, second specific antibody whereas for homodimers a stoichiometric hindering of two closely adjacent antibodies has to be considered.

In general the data demonstrate that EGFRvIII is also organized in clusters as it has been shown recently for wt EGFR. ${ }^{9,12}$ Moreover, the cluster size appears to be comparable to wt $\mathrm{EGFR}^{9}$ which, however, may be a general value for cluster formation since ErbB2 receptors have shown similar size distri- butions. ${ }^{10}$ This might be due to the physical properties of membranes and their tendency to form clusters because of entropic and hydrophobic forces. ${ }^{4-8}$

In order to verify our cluster analysis procedure and to exclude that the EGFRvIII clusters are only a result of image processing procedures, the EGFRwt receptors were analysed on BS153vIII+ and BS153vIII- cells according to the same procedures as the EGFRvIII receptors. The analyses (Table 2) result in cluster organization of EGFRwt which is in agreement with the established literature. ${ }^{12}$ 
(a)

(a)

(c)

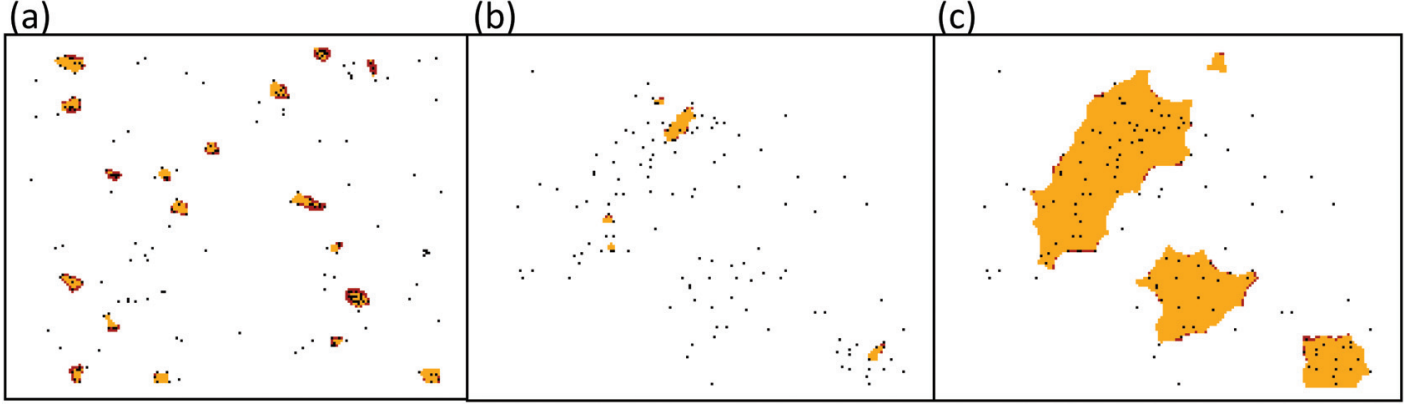

Fig. 5 Examples for cluster definition according to different parameters. (a) Clusters (yellow) of receptors (points) of a DKMGvlll+ cell section according to the parameter 5 next neighbours within a radius of $60 \mathrm{~nm}$ (used for evaluation); (b) clusters (yellow) of receptors (points) of a DKMGvIII- cell section according to the parameter 5 next neighbours within a radius of $60 \mathrm{~nm}$ (used for evaluation); (c) for comparison clusters (yellow) of receptors (points) of a DKMGvlll- cell section according to the parameter 5 next neighbours within a radius of $130 \mathrm{~nm}$.
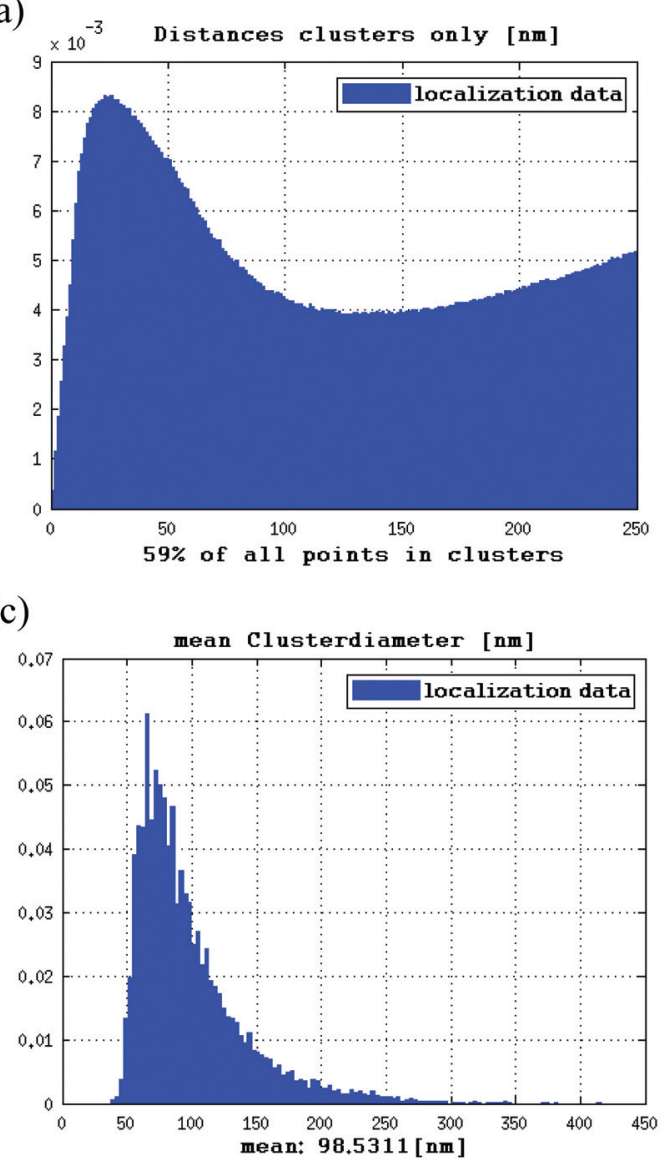

(b)

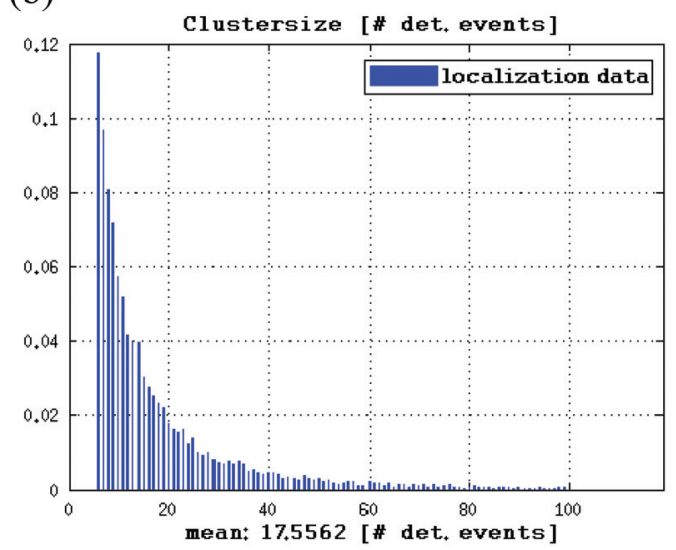

(d)

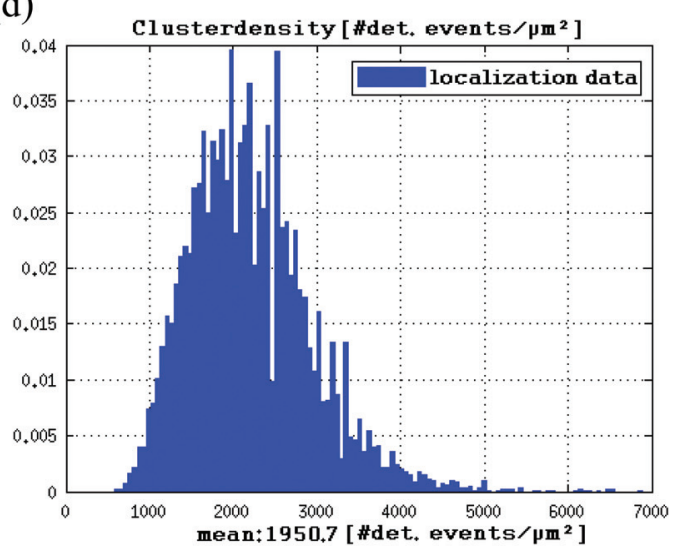

Fig. 6 Examples of frequency histograms for the cluster analysis (here for DKMGvlll+ cells): (a) distances in clusters (in nm), (b) cluster sizes (= number of points detected in the individual clusters), (c) cluster diameters (approximated by a circle in nm), (d) point densities in the clusters (detected points per $\mu \mathrm{m}^{2}$ ).

The two EGFRvIII+ cell lines showed differences in the degree of overexpression (Fig. 1) which seems to be reflected by the differences in the cluster arrangements while the EGFRvIII- cell lines did not show these differences. Comparing the controls no significant differences were found in the EGFRvIII- cell lines. Since the EGFRvIII- cells were specifically labelled for EGFRvIII and the physical membrane effects should also occur, one could conclude that the EGFRvIII- cell lines like the controls do not have any specific binding sites and show unspecific attachment only. On the other hand one has to consider that compared to feasible scaling of the measurements in the ten nanometre range, the 
Table 1 Results of the cluster analysis (EGFRvIll labelling)

\begin{tabular}{|c|c|c|c|c|c|}
\hline vIII labelling & $\begin{array}{l}\text { Percentage of signals } \\
\text { found in clusters [\%] }\end{array}$ & $\begin{array}{l}\text { Mean cluster size } \\
\text { [number of signals] }\end{array}$ & $\begin{array}{l}\text { Peak position in the } \\
\text { distance histogram [nm] }\end{array}$ & $\begin{array}{l}\text { Mean diameter } \\
\text { of clusters }[\mathrm{nm}]\end{array}$ & $\begin{array}{l}\text { Mean signal density } \\
{\left[1000 \mathrm{\mu m}^{-2}\right]}\end{array}$ \\
\hline BS153vIII+ & 67 & $22.4 \pm 4.8$ & $45 \pm 5$ & $116.8 \pm 10.9$ & $1.74 \pm 0.70$ \\
\hline BS153vIII + control & 8 & $9.5 \pm 3.1$ & $25 \pm 5$ & $69.3 \pm 8.4$ & $2.38 \pm 0.96$ \\
\hline BS153vIII- & 14 & $10.0 \pm 3.2$ & $20 \pm 5$ & $71.6 \pm 8.5$ & $2.32 \pm 0.93$ \\
\hline BS153vIII - control & 13 & $9.7 \pm 3.2$ & $25 \pm 5$ & $71.4 \pm 8.5$ & $2.25 \pm 0.90$ \\
\hline DKMGvIII+ & 59 & $17.6 \pm 4.2$ & $25 \pm 5$ & $98.5 \pm 10.0$ & $1.95 \pm 0.78$ \\
\hline DKMGvIII + control & 10 & $9.3 \pm 3.1$ & $25 \pm 5$ & $68.6 \pm 8.3$ & $2.45 \pm 0.98$ \\
\hline DKMGvIII- & 21 & $11.4 \pm 3.4$ & $25 \pm 5$ & $74.4 \pm 8.7$ & $2.36 \pm 0.95$ \\
\hline DKMGvIII - control & 10 & $9.4 \pm 3.1$ & $25 \pm 5$ & $70.4 \pm 8.4$ & $2.23 \pm 0.90$ \\
\hline
\end{tabular}

Table 2 Results of the cluster analysis (EGFRwt labelling)

\begin{tabular}{llllll}
\hline wt labelling & $\begin{array}{l}\text { Percentage of signals } \\
\text { found in clusters [\%] }\end{array}$ & $\begin{array}{l}\text { Mean cluster size } \\
{[\text { number of signals }]}\end{array}$ & $\begin{array}{l}\text { Peak position in the } \\
\text { distance histogram }[\mathrm{nm}]\end{array}$ & $\begin{array}{l}\text { Mean diameter } \\
\text { of clusters }[\mathrm{nm}]\end{array}$ & $\begin{array}{l}\text { Mean signal density } \\
{\left[1000 \mu \mathrm{m}^{-2}\right]}\end{array}$ \\
\hline BS153vIII+ & 36 & $12.6 \pm 3.5$ & $28 \pm 5$ & $90.6 \pm 9.5$ & $1.9 \pm 1.4$ \\
BS153vIII + control & 8 & $8.3 \pm 2.9$ & $22 \pm 5$ & $66.6 \pm 8.2$ & $2.4 \pm 1.1$ \\
BS153vIII- & 8 & $10.7 \pm 3.3$ & $28 \pm 5$ & $76.4 \pm 8.7$ & $2.3 \pm 1.4$ \\
BS153vIII - control & 6 & $11.1 \pm 3.3$ & $33 \pm 5$ & $71.2 \pm 8.4$ & $2.7 \pm 1.3$
\end{tabular}

labelling scheme consisting of primary and secondary antibodies requires a minimum space due to stoichiometric orientation which may be in the range of $25 \mathrm{~nm}$. Therefore specific antibodies directly tagged by fluorochromes should be used for future experiments.

\section{Conclusion}

Based on the EGFRvIII expressing glioblastoma cell lines BS153 and DKMG, we have recently established two pairs of iso-genetic sublines with either a high amount of EGFRvIII expressing cells (BS153vIII+ and DKMGvIII+) or a low amount of EGFRvIII expressing cells (BS153vIII- and DKMGvIII-). ${ }^{21}$ Although simulation experiments in theoretical biophysics have predicted cluster formation as a general feature of transmembrane receptors, ${ }^{4-8}$ it has been under debate whether EGFRvIII receptors form clusters in tumour cells or not. We have analysed these glioblastoma sublines by high precision localization microscopy. ${ }^{22}$ We were able to verify the cluster formation and to further quantify the clusters with reference to EGFRvIII expression. In summary approximately $60 \%$ of EGFRvIII is localized in clusters with about $100 \mathrm{~nm}$ cluster size on average which is in the range of lipid rafts. The analysis of the clusters of the EGFRvIII+ cell lines in comparison to the EGFRvIII- cell lines concerning size and signal point density may support assumptions of a functional dependent receptor distance change as already described for other members of the EGF-receptor family, e.g. ErbB2 and ErbB3 (see ref. 43 and 46 and our own unpublished data). Here it may be correlated to EGFRvIII dimerization as being known for wt EGFR receptors as well as for other receptors of the Erb-family. So far insights into the structural behaviour for receptor dimerization have been either obtained by transmission electron microscopy or crystallographic analysis, both requiring sophisticated pro- cedures of preparation. These shortcomings may be circumvented in many cases by localization microscopy maintaining the natural environment of the membranes by proceeding single molecule analysis. Using this potential of localization microscopy to maintain the intact 3D cell morphology will allow the study of the impact of EGFRvIII clustering on wt EGFR clustering as well as the formation of dimers after application of an appropriate labelling scheme. Since cluster formation already turned out to have an important impact on receptor signalling, such studies will provide valuable new insights into the biology of the tumour. Based on the investigation presented here future investigations on mechanisms associated with EGFRvIII receptor activation might help to improve anti-EGFRvIII therapy in GBM patients.

\section{Experimental section}

\section{Cell culture}

BS153vIII+/- and DKMGvIII+/- sublines have recently been described in detail. ${ }^{21}$ BS153 sublines were cultured in DMEM (Sigma-Aldrich) supplemented with $10 \%$ heat inactivated FCS, $2 \mathrm{mM}$ L-glutamine and $1 \mathrm{mM}$ sodium pyruvate (SigmaAldrich); DKMG cells were cultured in RPMI (10\% heat inactivated FCS, $2 \mathrm{mM}$ L-glutamine, $1 \mathrm{mM}$ sodium pyruvate). All cells were grown at $37{ }^{\circ} \mathrm{C}, 5 \% \mathrm{CO}_{2}$ and $100 \%$ humidification. All cells were identified by a short tandem repeat multiplex assay (Applied Biosystems).

\section{Flow cytometry}

Viable cells were incubated in phosphate buffered saline containing $3 \mathrm{mM}$ EDTA for $15 \mathrm{~min}$ at $37^{\circ} \mathrm{C}$ and then detached by scraping. An incubation was carried out with the anti EGFRvIII antibody L8A4 (mouse; kindly provided by Dr. Bigner; IgG, $1 \mu \mathrm{g} \mathrm{mL}{ }^{-1}$ ) for $1.5 \mathrm{~h}$ at $4{ }^{\circ} \mathrm{C}$. The cells were then washed two 
times with PBS/EDTA and exposed to a secondary Alexa fluor ${ }^{\circledR}$ 647 antibody (Molecular Probes) for $1 \mathrm{~h}$ at $4{ }^{\circ} \mathrm{C}$. After additional washing samples with and without (= control) a primary antibody were analysed by means of flow cytometry (FACScan Canto, BD Biosciences) to determine total EGFRvIII expression. Side scatter signals vs. red fluorescence were measured. Two windows were separated in the final dot-blots referring to EGFRvIII positively and negatively labelled cells.

\section{Western blotting}

Proteins from whole cell extracts were detected by western blotting according to standard protocols (see for instance ref. 44). The EGFR antibody was commercially obtained from Cell Signaling Technology while the anti $\beta$-actin antibody was purchased from Sigma-Aldrich. Secondary anti-mouse and antirabbit antibodies were purchased from LI-COR Biosciences. The Odyssey® CLx Infrared Imaging System (LI-COR Biosciences) was used for signal detection.

\section{Specimen preparation and immunofluorescence staining for 3D- and localization microscopy}

For localization imaging the cells were cultivated on round cover glasses in a 12 -well plate at $37{ }^{\circ} \mathrm{C}$. After $24 \mathrm{~h}$ the cells were washed in $2 \times$ PBS and fixed in $4 \%$ formaldehyde (prepared from paraformaldehyde). After blocking in $1 \times$ PBS and $3 \%$ BSA for $1 \mathrm{~h}$ at RT the cells were incubated with the anti EGFRvIII antibody (L8A4, mouse IgG, $1 \mu \mathrm{g} \mathrm{mL} \mathrm{m}^{-1}$ in $3 \%$ PBS/ BSA) or with the EGFRwt antibody (Santa Cruz \#sc-101, mouse IgG) for $1 \mathrm{~h}$. After washing three times with $1 \times \mathrm{PBS} / 0.5 \%$ Tween 20 the specimens were incubated with the ALEXAfluor ${ }^{\circledR}$ 594 labeled secondary antibody (in 3\% BSA, ALEXAfluor ${ }^{\circledR} 594$ anti-mouse antibody 1:1000; Molecular Probes or Thermo Fisher Scientific) for $1 \mathrm{~h}$ in the dark followed by washing three times in $1 \times \mathrm{PBS} / 0.5 \%$ Tween 20 and counter-stained with DAPI $(1: 1000)$. The specimens were embedded in ProlongGold antifade medium and sealed with nail polish. Until image acquisition the specimens were stored at $4{ }^{\circ} \mathrm{C}$ in the dark.

\section{D-Microscopy}

For 3D-imaging a Zeiss Axioplan 2 fluorescence microscope equipped with an Apotome scanning unit was used. An objective lens $63 \times /$ NA 1.4 was applied in combination with appropriate filter settings for the staining used. From the 3D-images maximum projection images were used for further evaluation.

\section{Localization microscopy}

For localization microscopy a setup has been used that combines structured illumination (ref. 45; not used in this application) and single molecule localization microscopy. ${ }^{41}$ It is an epifluorescence microscope that can change between two separate light paths, one at a high intensity density $\left(10 \mathrm{~kW} \mathrm{~cm} \mathrm{~cm}^{-2}\right)$ and the other at a lower one $\left(0.2 \mathrm{~kW} \mathrm{~cm}^{-2}\right)$. The former is used in the localization modus applied here. One can switch between different solid state lasers of wavelengths $488 \mathrm{~nm}$ and $568 \mathrm{~nm}$. Their power was set to $200 \mathrm{~mW}$ which corresponds to a power density of $10 \mathrm{~kW} \mathrm{~cm} \mathrm{~cm}^{-2}$ during localization data acqui- sition. The intensity of the laser(s) can be regulated via a neutral density filter wheel (i.e. a neutral grey filter) in up to 12 steps. Movable mirrors can switch it between the two modes.

The emitted photons are detected by using a high quantum efficiency CCD camera (Sensicam QE, PCO, Kelheim, Germany) after passing through a dichroic filter-wheel and a blocking filter-wheel (also called emission filter-wheel). The camera has a very sensitive and fast CCD-chip consisting of an array of $1376 \times 1040$ pixels with an area of $6.45 \times 6.45 \mu^{2}$ for each pixel. Together with the objective $100 \times / \mathrm{NA} 1.4$, this results in a pixel size of $64.5 \times 64.5 \mathrm{~nm}^{2}$ for the raw data.

\section{Data acquisition}

Cells were selected by visual inspection. For acquisition of image stacks, a Region Of Interest (ROI) was set in such a way that the ROIs completely covered the plasma membrane. The areas of the cell nucleus were omitted from the ROI, because the membrane was expected to be in another focal plane there. After determination of a ROI, the wide-field images were taken. Once all wide-field pictures were taken, the same had to be done for the localization recording. Depending on the specimen quality the image stacks were acquired with an integration time of typically $50 \mathrm{~ms}$ to $200 \mathrm{~ms}$ and a number of images between 500 and 2000. Usually 1000 images at $100 \mathrm{~ms}$ were chosen for comparison of the measurements.

\section{Data processing and cluster analysis}

In order to avoid preparation effects of cells from the same specimen, only cells with a number of detected points approaching an approx. 10\% range around the average point number of the same specimen were considered for further evaluation. Thus, variations only due to labelling efficiency could be avoided. Based on this pre-selection 25 cells per cell line were evaluated.

For further processing various MATLAB-scripts were used to identify the point positions and intensities of the single signals and to save them in a so-called "orte-matrix". In order to obtain this, the program requires the conversion factor from pixels to nm. The orte-matrix consists of nine columns: (a) the amplitude of the signal in photoelectrons, (b) the lateral $y$-coordinate in $\mathrm{nm},(\mathrm{c})$ the lateral $x$-coordinate in $\mathrm{nm},(\mathrm{d}, \mathrm{e})$ the measurement errors for $x$ and $y$ coordinates, $(\mathrm{f}, \mathrm{g})$ the standard deviations, (h) the number of photoelectrons in the signal i.e. counts and (i) the number of the image in which the signal is found.

The exactness of the determination of the point positions is called localization precision. It is influenced by many factors, such as instrumental setup, wavelength, fluorophore type, sample type, sample preparation and embedding medium, so that in practice the localization precision is far below the theoretical limits of the instrument. The cluster analysis applied here uses the single fluorophore positions. These were determined for each time stack of a cell; an example is shown in Fig. 7.

Further calculations were carried out on the basis of the orte-matrix, for example the localization image, the nearest 


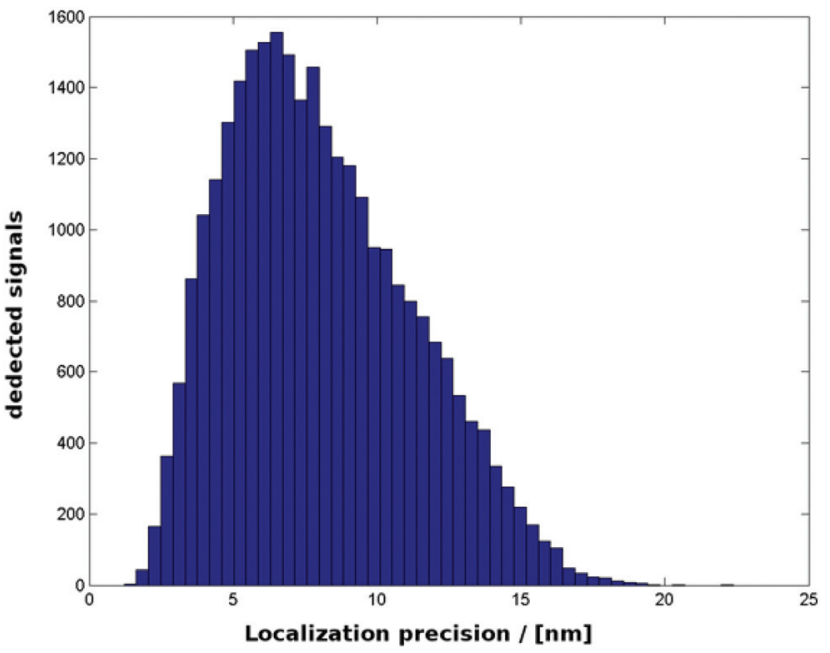

Fig. 7 Example of the measurements of localization precision of one cell. The frequencies of the full widths at half maximum of the detected intensity distributions of each point are shown. The peak position determines the localization precision of the image of this cell.

neighbour image or the cluster analysis. In the localization image created from the orte-matrix, the brightness of a point determines the localization precision, i.e. the brighter a point appears the more precisely it is identified. In the nearestneighbour image (NN-image), the brightness indicates how many signals are neighbouring a certain signal.

In order to study receptor clustering the evaluation algorithm autoclusters_v $3^{10,40}$ was used. After iterative exclusion (see the results), a cluster radius of $60 \mathrm{~nm}$ and a minimum number $N=5$ neighbours within this radius were set to determine the minimum conditions for a cluster point. The cluster radius then extended from a point, which was consistent with the minimum number of $N$ within the required radius. This means that points that lay within this defined cluster radius were included into one cluster and all the other points were excluded. From an image analysed in such a way, distance and cluster distributions, point densities, cluster sizes (number of counts in a cluster), cluster diameters etc. were calculated.

For computation of the point density, a circle with a radius of $400 \mathrm{~nm}$ was set around each point. This radius of $400 \mathrm{~nm}$ was empirically determined and was independent from the cluster radius. Since the ROI may also contain some non-cellular areas, the area of all circles together gives a precise approximation of the real cell area, at least for cells with a nearly homogeneous point distribution. The quotient of the number of points and the area was defined as the point density. The cluster density was calculated by the inverse square of the average NN-distances.

\section{Funding}

N. Struve, M. Kriegs: City of Hamburg, Landesexzellenzinitiative Hamburg (LEXI 2012; Tumor target- ing via cell surface molecules essential in cancer progression and dissemination); M. Kriegs: BMBF (02NUK032); Hamburger-Krebsgesellschaft; Brigitte and Dr. Konstanze Wegener-Stiftung; Erich und Gertrud Roggenbuck-Stiftung; Forschungsförderung der medizinischen Fakultät am UKE, Hamburg; Spierling Stiftung.

\section{Acknowledgements}

The authors thank Dr. Jutta Schwarz-Finsterle, Dr. Patrick Müller, and Michael Stuhlmüller for their continuous help and fruitful discussion. Finally the authors thank Dr. CorinnaCornelia Seegler-Sandbanck, the European Institute for Feasibility Studies, for bringing light into dark moments of science.

\section{References}

1 B. F. El-Rayes and P. M. LoRusso, Br. J. Cancer, 2004, 91, 418-424.

2 E. J. Bernhard, E. J. Stanbridge, S. Gupta, A. K. Gupta, D. Soto, V. J. Bakanauskas, G. J. Cerniglia, R. J. Muschel and W. G. McKenna, Cancer Res., 2000, 60, 6597-6600.

3 P. Maier, L. Hartmann, F. Wenz and C. Herskind, Int. J. Mol. Sci., 2016, 17, 102.

4 D. Morozova, G. Guigas and M. Weiss, PLoS Comput. Biol., 2011, 7, e1002067.

5 G. Guigas and M. Weiss, Soft Matter, 2015, 11, 33-37.

6 U. Schmidt, G. Guigas and M. Weiss, Phys. Rev. Lett., 2008, 101, 128104.

7 G. Guigas and M. Weiss, Biophys. J., 2008, 95, L25-L27.

8 U. Schmidt and M. Weiss, Biophys. Chem., 2010, 151, 34-38.

9 A. Abulrob, Z. Lu, E. Baumann, D. Vobornik, R. Taylor, D. Stanimirovic and L. J. Johnston, J. Biol. Chem., 2010, 285, 3145-3156.

10 R. Kaufmann, P. Müller, G. Hildenbrand, M. Hausmann and C. Cremer, J. Microsc., 2011, 242, 46-54.

11 Y. Wang, J. Gao, X. Gao, T. Tong, X. Shi, L. Li, M. Qi, Y. Wang, M. Cai, J. Jiang, H. Ji and H. Wang, Cell Res., 2014, 24, 959-976.

12 J. Gao, Y. Wang, M. Cai, Y. Pan, H. Xu, J. Jiang, H. Ji and H. Wang, Nanoscale, 2015, 7, 2511.

13 D. J. Irvine, K. A. Hue, A. M. Mayes and L. G. Griffith, Biophys. J., 2002, 80, 120-132.

14 H. K. Gan, A. N. Cvrijevic and T. G. Johns, FEBS J., 2013, 280, 5350-5370.

15 R. Stupp, M. E. Hegi, W. P. Mason, M. J. van den Bent, M. J. Taphoorn, R. C. Janzer, S. K. Ludwin, A. Allgeier, B. Fisher, K. Belanger, P. Hau, A. A. Brandes, J. Gijtenbeek, C. Marosi, C. J. Vecht, K. Mokhtari, P. Wesseling, S. Villa, E. Eisenhauer, T. Gorlia, M. Weller, D. Lacombe, J. G. Cairncross and R. O. Mirimanoff, Lancet Oncol., 2009, 10, 459-466. 
16 C. Adamson, O. O. Kanu, A. I. Mehta, C. Di, N. Lin, A. K. Mattox and D. D. Bigner, Expert Opin. Invest. Drugs, 2009, 18, 1061-1083.

17 C. A. Del Vecchio, C. P. Giacomini, H. Vogel, K. C. Jensen, T. Florio, A. Merlo, J. R. Pollack and A. J. Wong, Oncogene, 2013, 32, 2670-2681.

18 A. J. Wong, M. Ruppert, S. H. Bigner, C. H. Grzeschik, P. A. Humphrey, D. S. Bigner and B. Vogelstein, Proc. Natl. Acad. Sci. U. S. A., 1992, 89, 2965-2969.

19 C. T. Chu, K. D. Everiss, C. J. Wikstrand, S. K. Batra, H. J. Kung and D. D. Bigner, Biochem. J., 1997, 324, 855861.

20 M. D. Marmor, K. B. Skaria and Y. Yarden, Int. J. Radiat. Oncol., Biol., Phys., 2004, 58, 903-913.

21 N. Struve, M. Riedel, A. Schulte, T. Rieckmann, T. J. Grob, A. Gal, K. Rothkamp, K. Lamszus, C. Petersen, E. Dikomey and M. Kriegs, Oncotarget, 2015, 6, 33867-33877.

22 C. Cremer, R. Kaufmann, M. Gunkel, S. Pres, Y. Weiland, P. Müller, T. Ruckelshausen, P. Lemmer, F. Geiger, M. Degenhard, C. Wege, N. Lemmermann, R. Holtappels, H. Strickfaden and M. Hausmann, Biotechnol. J., 2011, 6, 1037-1051.

23 E. Abbe, Arch. Mikrosk. Anat. Entwicklungsmech., 1873, 9, 411-468.

24 L. Rayleigh, Philos. Mag., 1896, 42, 167-195.

25 C. Cremer and B. Masters, Eur. Phys. J. H, 2013, 38, 281344.

26 A. Esa, P. Edelmann, G. Kreth, L. Trakhtenbrot, N. Amariglio, G. Rechavi, M. Hausmann and C. Cremer, J. Microsc., 2000, 199, 96-105.

27 E. Betzig, G. H. Patterson, R. Sougrat, O. W. Lindwasser, S. Olenych, J. Bonifacino, M. W. Davidson, J. LippincottSchwartz and H. F. Hess, Science, 2006, 313, 1642-1645.

28 S. T. Hess, T. P. Girirajan and M. D. Mason, Biophys. J., 2006, 91, 4258-4272.

29 M. J. Rust, M. Bates and X. Zhuang, Nat. Methods, 2006, 3, 793-795.

30 M. Heilemann, S. van de Linde, M. Schüttpelz, R. Kaspar, B. Seefeldt, A. Mukherjee, P. Tinnefeld and M. Sauer, Angew. Chem., Int. Ed., 2008, 47, 6172-6176.
31 P. Lemmer, M. Gunkel, D. Baddeley, R. Kaufmann, A. Urich, Y. Weiland, J. Reymann, P. Müller, M. Hausmann and C. Cremer, Appl. Phys. B, 2008, 93, 1-12.

32 J. Fölling, M. Bossi, H. Bock, R. Medda, C. Wurm, B. Hein, S. Jakobs, C. Eggeling and S. W. Hell, Nat. Methods, 2008, 5, 943-945.

33 S. Ram, E. S. Ward and R. J. Ober, Proc. Natl. Acad. Sci. U. S. A., 2006, 103, 4457-4462.

34 R. J. Ober, S. Ram and E. S. Ward, Biophys. J., 2004, 86, 1185-1200.

35 P. Lemmer, M. Gunkel, Y. Weiland, P. Müller, D. Baddeley, R. Kaufmann, A. Urich, H. Eipel, R. Amberger, M. Hausmann and C. Cremer, J. Microsc., 2009, 235, 163171.

36 J. Hendrix, C. Flors, P. Dedecker, J. Hofkens and Y. engelbotghs, Biophys. J., 2008, 94, 4103-4113.

37 D. Sinnecker, P. Voigt, N. Hellwig and M. Schaefer, Biochemistry, 2005, 44, 7085-7094.

38 Y. Zhang, G. Máté, P. Müller, S. Hillebrandt, M. Krufczik, M. Bach, R. Kaufmann, M. Hausmann and D. W. Heermann, PLoS One, 2015, 10, e0128555.

39 P. Müller, N. A. Lemmermann, R. Kaufmann, M. Gunkel, D. Paech, G. Hildenbrand, R. Holtappels, C. Cremer and M. Hausmann, Histochem. Cell Biol., 2014, 142, 61-67.

40 M. Stuhlmüller, J. Schwarz-Finsterle, E. Fey, J. Lux, M. Bach, C. Cremer, K. Hinderhofer, M. Hausmann and G. Hildenbrand, Nanoscale, 2015, 7, 17938-17946.

41 S. Rossberger, G. Best, D. Baddeley, R. Heintzmann, U. Birk, S. Dithmar and C. Cremer, J. Opt., 2013, 15, 094003.

42 C. J. Wikstrand, R. E. McLendon, A. H. Friedman and D. D. Bigner, Cancer Res., 1997, 57, 4130-4140.

43 P. Nagy, A. Janei, A. K. Kirsch, J. Szöllosi, S. Damjanovich and T. M. Jovin, J. Cell Sci., 1999, 112, 1733-1741.

44 T. Mahmood and P.-C. Yang, N. Am. J. Med. Sci., 2012, 4, 429-434.

45 M. G. L. Gustafsson, Proc. Natl. Acad. Sci. U. S. A., 2005, 102, 13081-13086.

46 S. Yang and M. A. Raymond-Stintz, J. Cell Sci., 2007, 120, 2763-2773. 